\title{
Nuclear myosin/actin-motored contact between homologous chromosomes is initiated by ATM kinase and homology-directed repair proteins at double-strand DNA breaks to suppress chromosome rearrangements
}

\author{
Viktoria N. Evdokimova ${ }^{1,}{ }^{*}$, Manoj Gandhi ${ }^{1,}{ }^{*}$, Alyaksandr V. Nikitski ${ }^{1}$, Christopher J. \\ Bakkenist $^{2}$ and Yuri E. Nikiforov ${ }^{1}$ \\ ${ }^{1}$ Department of Pathology, University of Pittsburgh, Pittsburgh, PA, 15213, USA \\ ${ }^{2}$ Department of Radiation Oncology and Pharmacology and Chemical Biology, University of Pittsburgh, Pittsburgh, PA, 15232, \\ USA \\ "These authors have contributed equally to this work \\ Correspondence to: Yuri E. Nikiforov, email: nikiforovye@upmc.edu \\ Keywords: chromosome; double-strand break; ATM; nuclear motors; DNA repair \\ Received: November 30, $2017 \quad$ Accepted: January 30, $2018 \quad$ Published: February 07, 2018 \\ Copyright: Evdokimova et al. This is an open-access article distributed under the terms of the Creative Commons Attribution \\ License 3.0 (CC BY 3.0), which permits unrestricted use, distribution, and reproduction in any medium, provided the original author \\ and source are credited.
}

\section{ABSTRACT}

We provide evidence for a mechanism of DNA repair that requires nuclear myosin/actin-dependent contact between homologous chromosomes to prevent formation of chromosomal rearrangement in human cells. We recently showed that DNA double strand breaks (DSBs) induced by $\mathrm{y}$-rays or endonucleases cause ATMdependent contact formation between homologous chromosomes at damaged sites of transcriptionally active chromatin in $\mathbf{G}_{0} / \mathbf{G}_{1}$-phase cells. Here, we report that the mechanism of contact generation between homologous chromosomes also requires homology-directed repair proteins, including BRCA1, RAD51 and RAD52, and nuclear myosin/actin-motors. Moreover, inhibition of ATM kinase or deficiency in nuclear actin polymerization causes carcinogenic RET/PTC chromosome rearrangements after DSBs induction in human cells. These data suggest that DSBs in transcriptionally active euchromatin in $G_{0} / G_{1}$-phase cells are repaired through a mechanism that requires contact formation between homologous chromosomes and that this mechanism is mediated by HDR proteins and nuclear myosin/actin motors.

\section{INTRODUCTION}

Mechanisms that ensure genome stability were essential for the origin of species and homeostasis in metazoans. DNA double-strand breaks (DSBs) which are induced by reactive oxygen radicals generated by oxidative phosphorylation and ionizing radiation (IR) are a particular hazard in mammalian cells as they are substrates for recombination events that can activate oncogenes leading to cancer. Two canonical DSB repair pathways exist in eukaryotic organisms. Non homologous end-joining (NHEJ) is an imperfect mechanism of repair wherein DSB ends are trimmed and then the ends are ligated together leading to a loss of genetic material at the site of the break. Homologous recombination is a mechanism of repair that uses a sister chromatid as a template to correct a DSB without loss of genetic material. Since this canonical mechanism of homologous recombination requires the co-localization of sister chromatids, it is generally associated with DNA replication forks in S phase or with G2 phase cells. However, observations that homologous chromosomes make contact after the induction of DSBs in $\mathrm{G}_{0} / \mathrm{G}_{1}$ cells, including mature thyroid cells [1,2], led us hypothesize that a mechanism of homologous recombination that uses a homologous chromosome as a template may exist in cells when there is no sister chromatid template. 
Several additional lines of evidence point to a mechanism that generates contact between homologous chromosomes after the induction of a DSB. Live cell imaging of diploid budding yeast revealed that endonuclease-induced DSB end in a red fluorescent protein-marked chromosome is rapidly repaired using the yellow fluorescent protein-marked homologous chromosome during S-phase [3]. Following DSB induction, the cleaved chromosome was observed moving through $\sim 30 \%$ of the nuclear volume, rather than $3 \%$ prior to DSB induction. Contact between homologous chromosomes was coincident with Rad52 foci that identify sites of strand exchange between chromatids or chromosomes. Homology-directed repair (HDR) was inferred from the observation that Rad52 foci disassembled before the homologous chromosomes separation. Increased mobility of an endonuclease-induced DSB end was also observed in haploid yeast [4]. The increased mobility of the DSB end required $\operatorname{Rad} 51$, a key recombinase protein that identifies sequence homology and pairs sister chromatids, Mec1, an apical DNA damage signaling kinase, and Rad9, a checkpoint protein. The increased mobility of the DSB end did not require the downstream kinase Rad53, suggesting that in yeast an apical DNA damage signaling kinase, but not checkpoint activation per se was required for the increased mobility.

In mammalian cells, increased mobility of $\gamma$-particle-induced DSB ends and uncapped telomeres were observed and this was dependent on 53BP1, the homologue of yeast $\operatorname{Rad} 9$ [5-7]. In contrast, limited mobility of DSB ends was observed after application of ultrasoft-X-ray or single endonuclease [8-10]. Allowing one explanation for this apparent discrepancy, we showed that contact between homologous chromosomes induced by either $\gamma$-rays or an endonuclease was limited to sites of DSBs that arose in transcriptionally active chromatin in human cells $[1,2]$. These data are generally supported by the finding that DSBs in transcriptionally active chromatin are preferentially repaired by homologous recombination in human cells [11]. However, factors required for homologous chromosomes mobility, their contact initiation or consequences of this failure remain largely unknown.

Increased mobility of endonuclease-induced DSB ends in telomeres in ALT (alternative lengthening of telomeres) positive $\mathrm{G}_{1}$-phase cancer cells has been reported [12]. The introduction of a DSB in an ALT-telomere induced RAD51-mediated telomere-telomere contact, which was associated with homology-directed telomere synthesis. DSBs induced in transcriptionally active rDNA repeats in nucleoli using either an endonuclease or CRISP/ Cas9 targeting increased the mobility of DSB ends of rDNA [13]. The introduction of a DSB in transcriptionally active rDNA repeats induced the migration of the site of the DSB from the nucleolar interior to anchoring points at the nucleolar periphery in $\mathrm{G}_{1^{-}}, \mathrm{S}$ - and $\mathrm{G}_{2}$-phase cells. The migration was ATM kinase-dependent and RAD51 foci colocalized with DSBs in rDNA at the nucleolar periphery. These data suggest that the mobility of ALT-telomere and nucleolar rDNA is increased after the induction of a DSB and that proteins required for homology-directed repair accumulate at these DSB ends in $\mathrm{G}_{1}$-phase cells. In addition to ATM and RAD51, the long-range directional movement of an interphase chromosome site may be dependent on nuclear myosin I (NMI) and actin nuclear motors [14].

Here, we show that contact between homologous chromosomes after induction of DSBs in transcriptionally active chromatin in $\mathrm{G}_{0 /} \mathrm{G}_{1}$-phase human cells requires homology-directed repair (HDR) but not non homologous end join (NHEJ) proteins. Furthermore, we demonstrate that it requires nuclear myosin/actin-motors. Finally, we show that ATM kinase inhibition or genetic inactivation of nuclear actin induce carcinogenic RET/PTC chromosome rearrangements in human cells.

\section{RESULTS}

\section{Contact between homologous chromosomes at sites of DSBs induced in genes requires HDR proteins}

Previously, we showed contact formation between homologous chromosomes at sites of DSBs in quiescent cells in transcriptionally active chromatin [2]. This contact formation required ATM, an apical DNA damage signaling kinase that is activated at DSBs and necessary for homology-directed DNA repair (HDR) pathways [15, 16]. We hypothesized that contact formation between homologous chromosomes after induction of DSBs in transcriptionally active chromatin also required HDR proteins.

To test this hypothesis, we generated human thyroid cancer TPC1/I-PpoI cells stably expressing HA-ER-I-PpoI fusion protein which translocates into nucleus and induces DNA DSBs at specific restriction sites upon induction with 4-hydroxytamoxifen (4-OHT) $[17,18]$. Expression of genes required for HDR (BRCA1, RAD51 and RAD52) and for NHEJ ( $K U 70, D N A-P K$ and $L I G I V$ ) was knocked down in TPC1/I-PpoI cells using siRNA (Supplementary Figure 1). I-PpoI-specific DSBs were then induced by treatment with 4-OHT, that was confirmed by $\gamma \mathrm{H} 2 \mathrm{AX}$ immunofluorescence. Frequency of contact formation between homologous chromosomes was analyzed at two I-PpoI-sensitive sites in the transcriptionally active $D A B 1$ and GRIP 1 genes in $\mathrm{G}_{0} / \mathrm{G}_{1}$-phase cells using $3 \mathrm{D}$ FISH (Figure 1A).

Our analysis revealed that frequency of contact between homologous chromosomes at DAB1 and GRIP1 genes upon induction of DSBs increased from baseline level of $2.6 \%$ to $7.7 \%$ and $7.8 \%$ in intact and scramble siRNA treated cells, respectively. This finding is concordant with 
previous data demonstrated that the I-PpoI restriction endonuclease cuts approximately $10 \%$ of sequence specific sites in a single cell $[2,19]$. When any of HDR genes (BRCA1, RAD51 and RAD52) expression was knocked down, the contact between homologous chromosomes was reduced to the baseline level. However, when the NHEJ genes were silenced, the frequency of I-PpoI-induced homologous chromosome contact was either unaffected after knocking down $D N A-P K$ and $K U 70$ or slightly reduced after knocking down $L I G I V$ (Figure 1B).

Thus, the HDR factors BRCA1, RAD51 and RAD52, but not NHEJ factors DNA-PK and KU70 are required for DSBs-induced contacts formation between homologous chromosomes at the transcribed $D A B 1$ and GRIPl genes in $\mathrm{G}_{0} / \mathrm{G}_{1}$-phase cells.

\section{Rad51 co-localizes with sites of contact between homologous chromosomes at DSBs induced in genes in $\mathbf{G}_{0} / \mathbf{G}_{1}$-phase cells}

To further confirm the role of HDR factors in contact formation between homologous chromosomes after induction of DSBs, we performed 3D immunoFISH with DNA probes for the I-PpoI-sensitive regions of $D A B 1$ gene and intergenic $5 \mathrm{qIG}$ locus or for the I-PpoIinsensitive $16 \mathrm{pNC}$ non-coding region. Additionally,

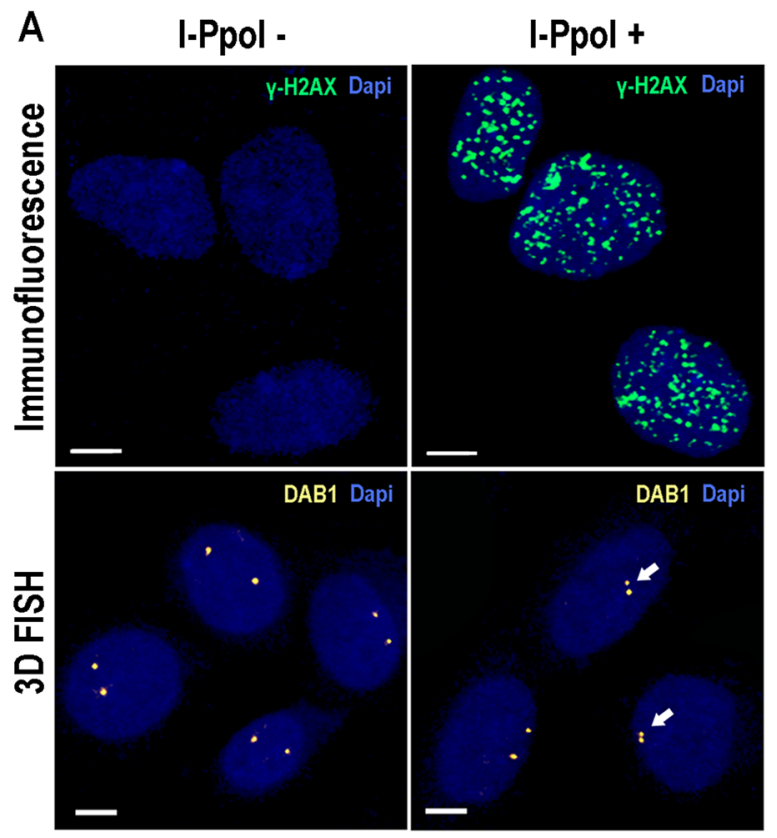

B

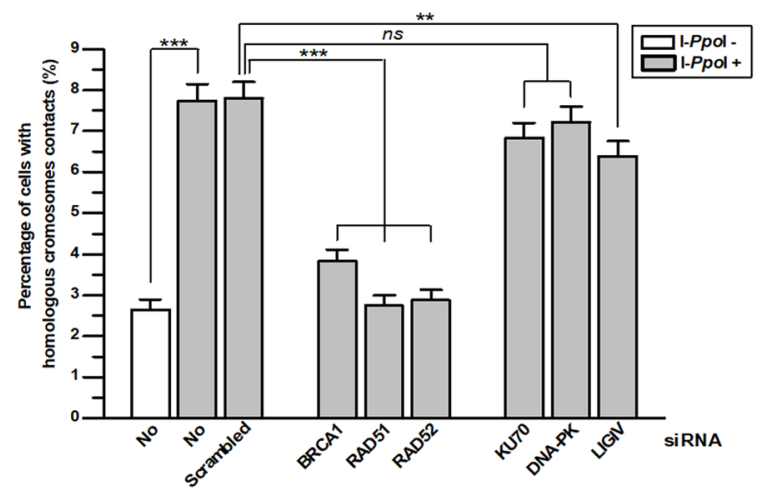

Figure 1: Contact formation between homologous chromosomes at DSBs in DAB1 and GRIP1 genes requires HDR proteins. TPC1/I-PpoI cells were treated with 4-OHT for $6 \mathrm{~h}$ to induce translocation of the ER-I-PpoI fusion protein into the nucleus and generate DSBs at specific sites. (A) Efficiency of DSB induction by I-PpoI in TPC1/I-PpoI cells was confirmed by immunofluorescence with anti- $\gamma \mathrm{H} 2 \mathrm{AX}$ antibodies. The intranuclear localization of I-PpoI-sensitive $D A B 1$ gene regions of homologous chromosomes without and after induction of DSBs by I-PpoI was determined with 3D FISH. Arrows identify contact formation between homologous chromosomes. Scale bar - $5 \mu \mathrm{m}$. (B) Graph showing the increase in contact formation between homologous chromosomes at DAB1 and GRIP1 genes after induction of DSBs by I-PpoI. Knockdown of HDR proteins (BRCA1, RAD51 and RAD52) by siRNA abolished contact formation between homologous chromosomes. Data for $D A B 1$ and GRIP1 regions are combined and presented as mean $\pm \mathrm{SEM} ;{ }^{* *}, P<0.001 ;{ }^{* * *}, P<0.0001$. 
antibodies against RAD51 and PCNA were used. Only $\mathrm{G}_{0} / \mathrm{G}_{1}$-phase cells showing diffuse/homogenous PCNA staining were analyzed. 3D immuno-FISH showed that after induction of DSBs, RAD51 was co-localized with $43 \%$ of contacts between homologous chromosomes at $D A B 1$ gene (Figure 2). RAD51 foci were not expected in $100 \%$ of contacts since part of homologous chromosomes might be in contact after the dissociation of the RAD51 filaments. Regardless of the chromosome contact, RAD51 foci were co-localized with the transcriptionally active $D A B 1$ gene in $11.7 \%$ of $\mathrm{G}_{0} / \mathrm{G}_{1}$-phase cells. In contrast, RAD51 was co-localized with I-PpoI-sensitive intergenic $5 \mathrm{qIG}$ locus only in $1.7 \%$ of cells (Figure 2C, 2D). This was similar to the baseline level of spontaneous RAD51 foci co-localization with anti-16pNC FISH probes (Figure 2D). These data reveal that RAD51 co-localizes
A

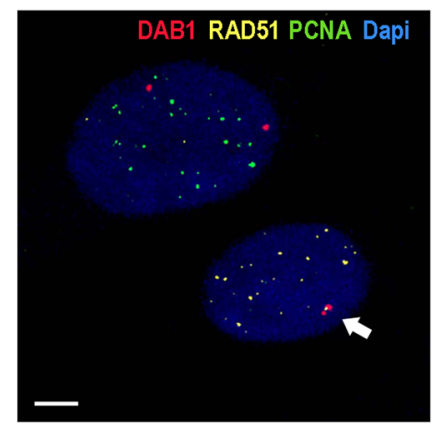

C

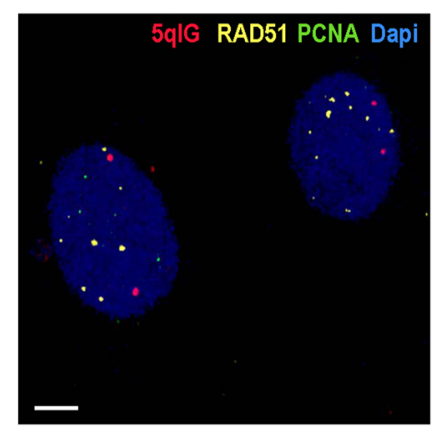

D

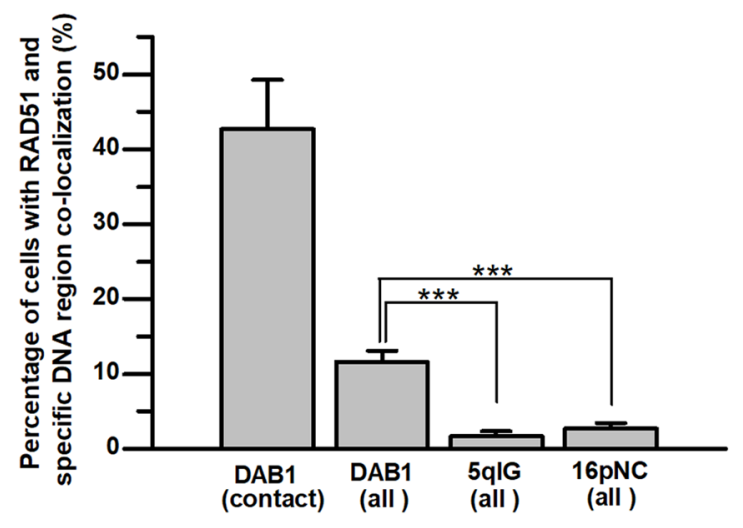

B

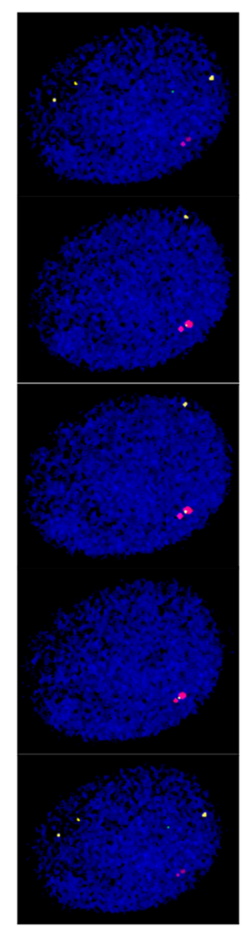

(all)

Figure 2: Co-localization of RAD51 with sites of contact between homologous chromosomes after induction of DSBs in $\mathbf{G}_{\mathbf{0}} / \mathbf{G}_{\mathbf{1}}$-phase cells. TPC1/ I-PpoI cells were treated with 4-OHT for 6 hours prior to 3D immuno-FISH analysis. (A) Representative 3D immuno-FISH confocal image showing co-localization of RAD51 with homologous chromosomes contact (arrow) at transcriptionally active $D A B 1$ gene in $\mathrm{G}_{0} / \mathrm{G}_{1}$-phase cells (lower nucleus). S-phase cells with characteristic focal pattern of PCNA staining (upper nucleus) were excluded from analysis. (B) 3-D co-localization of the RAD51 with DAB1 signals was confirmed by sequential analysis of the Z-stacked images. (C) 3D immuno-FISH with probe for the I-PpoI-sensitive 5qIG intergenic region did not reveal notable co-localization of 5qIG and RAD51 signals. Scale bar - $5 \mu \mathrm{m}$. (D) Graph showing higher frequencies of RAD51 co-localization with transcriptionally active $D A B 1$ gene in $\mathrm{G}_{0} / \mathrm{G}_{1}$-phase cells, especially at sites of inter-chromosomal contacts, compared to the silent intergenic $5 \mathrm{qIG}$ or I-PpoI -insensitive $16 \mathrm{pNC}$ chromosomal regions. Data are presented as mean $\pm \mathrm{SEM} ;{ }^{* * *}, P<0.0001$. 
with contacts between homologous chromosomes at sites of DSBs induced in transcriptionally active chromatin in $\mathrm{G}_{0} / \mathrm{G}_{1}$-phase cells.

\section{Contact between homologous chromosomes at sites of DSBs induced in genes in $\mathbf{G}_{0} / \mathbf{G}_{1}$-phase cells requires nuclear myosin $I$ and actin nuclear motors}

Based on the previous findings implicating NMI and actin in the directional movements of interphase chromosomes, we investigated the role of these proteins in contact formation between homologous chromosomes at sites of DSBs induced in genes. For this, NMI was knocked down in TPC-1/I-PpoI cells by treatment with specific siRNA (Supplementary Figure 1). Alternatively, the function of actin was blocked using two drugs that affect F-actin polymerization, jasplakinolide [20] and latrunculin B [21].

As shown before, contacts between homologous chromosomes at sites of DSBs induced in the $D A B 1$ and GRIP1 genes were identified in $7.4 \%$ and $7.8 \%$ of intact and scramble siRNA treated cells, respectively. Upon knock-down of NMI, the frequency of the contact between homologous chromosomes decreased to $3.2 \%$, close to the baseline observed without DSBs induction. Similar decrease was revealed after treatment with jasplakinolide and latrunculin B (Figure 3A).

To further explore the role of nuclear motors in homologous chromosomes contact formation, we transfected TPC-1/I-PpoI cells with expression plasmids for wild-type actin, G13R-mutant actin that is deficient in F-actin polymerization, and S14C-mutant actin that in opposite increases F-actin polymerization [14, 22]. In the absence of DSBs-induction, contacts between homologous chromosomes at the DAB1 and GRIP1 genes were identified in 3.5-3.7\% of cells expressing different types of actin. However, I-PpoI-induced homologous chromosome contact at DAB1 and GRIP1 was identified in $7.0 \%$ of cells expressing wild-type actin; in $3.6 \%$ of cells expressing G13R-mutant actin that is deficient in actin polymerization; and in $11.0 \%$ of cells expressing S14C-mutant actin that increases F-actin polymerization (Figure 3B). Thus, three different experimental approaches demonstrate that NMI and proper polymerization of F-actin are required for contact formation between homologous chromosomes at the sites of DSBs in active genes in $\mathrm{G}_{0} / \mathrm{G}_{1}$-phase cells.

\section{ATM kinase activity and F-actin polymerization are required for chromosome stability after the induction of DSBs}

Since ATM kinase activity and myosin/actin nuclear motors are required for contact between homologous chromosomes at DSBs in transcriptionally active chromatin, we hypothesized that inhibition of ATM kinase or F-actin polymerization would prevent the formation of homologous chromosomes contact at sites of DSBs, thereby promoting breaks misrepair and formation of
A

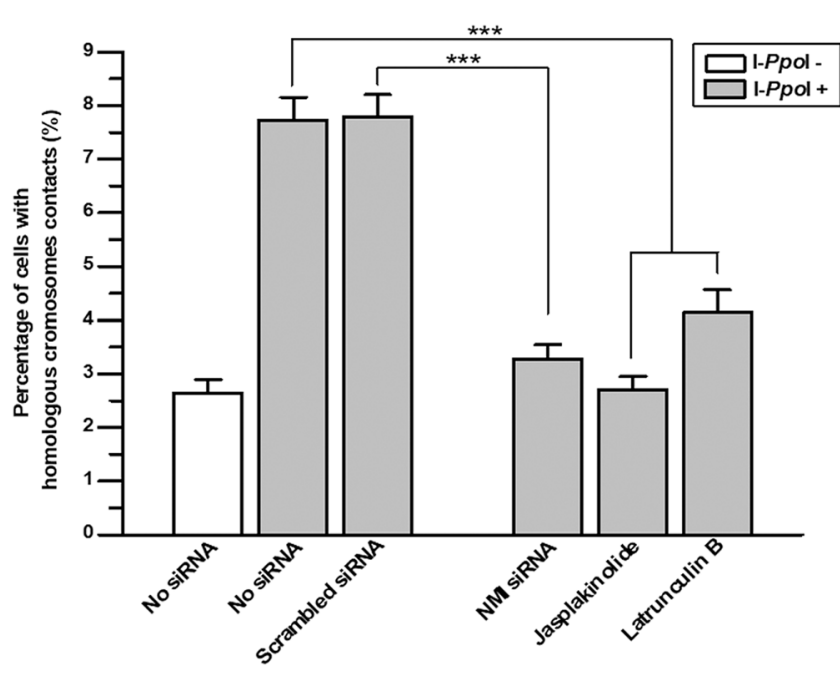

B

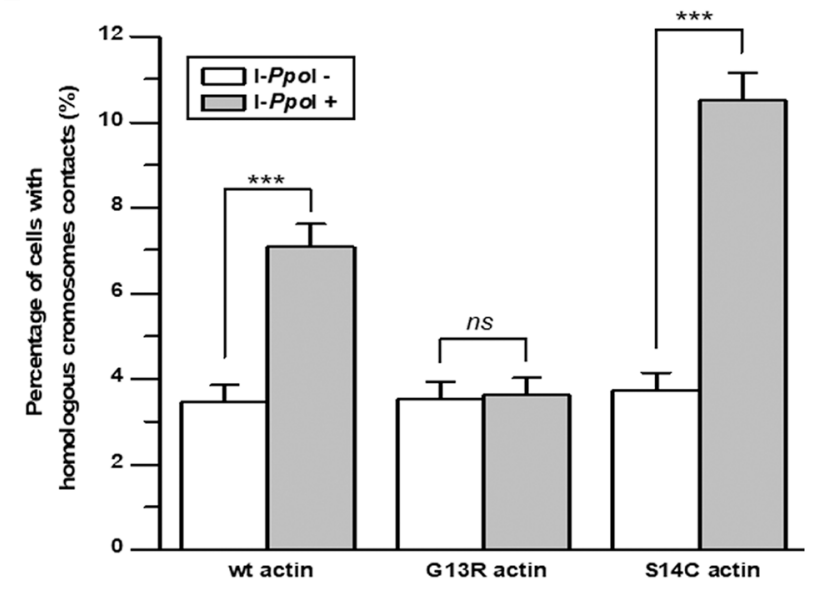

Figure 3: Nuclear myosin I and actin are required for contact formation between homologous chromosomes at sites of DSBs in $\boldsymbol{D A B} 1$ and $\boldsymbol{G R I P 1}$ genes. (A) Knockdown of NMI using siRNA for $72 \mathrm{~h}$ or disruption of F-actin polymerization with jasplakinolide or latrunculin B for $2 \mathrm{~h}$ decreased the frequency of contact formation between homologous chromosomes at DSBs in DAB1 and GRIP1 genes. (B) Graph showing that polymerization deficiency of G13R-mutant actin reduced the frequency of contact formation between homologous chromosomes. In contrast, S14C-actin, which promotes filament polymerization, increased contact formation between DSBs sites of homologous chromosomes. Data are presented as mean $\pm \mathrm{SEM} ;{ }^{* * *}, P<0.0001$. 
chromosome rearrangements. To test this hypothesis, we used an assay that detects the formation of endonucleaseinduced $R E T / P T C$ rearrangements [23, 24]. Analyzed RET/PTC1 and RET/PTC3 are intrachromosomal inversions involving the RET and CCDC6 or NCOA4 genes, respectively, which are prevalent in thyroid cancers in individuals exposed to radiation after the Chernobyl nuclear accident [25]. Importantly, RET/PTC can be induced in $\mathrm{G}_{0} / \mathrm{G}_{1}$-phase thyroid cells in vivo by exposure to ionizing radiation or restriction endonucleases [23, 24]. The endonuclease PvuII induces DSBs in intron 11 of the $R E T$ gene, which lead to the formation of RET/PTC in HTori-3 human thyroid cell line [24].

First, we investigated how ATM kinase inhibition impacts the endonuclease-induced RET/PTC rearrangements. HTori-3 cells were electroporated with PvuII restriction enzyme and treated with the ATM inhibitor KU55933. DSB induction by PvuII was assessed by $\gamma \mathrm{H} 2 \mathrm{AX}$ immunofluorescence and $75-82 \%$ of cells electroporated with PvuII were positive for DNA damage, as compared to $5-6 \%$ of control cells (data not shown). PvuII-induced RET/PTC were observed in $2.4 \pm 0.4$ cells per million in vehicle control, and this increased to $5.3 \pm 0.5$ cells per million with ATM kinase inhibitor. Surprisingly, in the absence of PvuII, RET/PTC rearrangements were observed in $0.3 \pm 0.3$ cells per million with ATM kinase inhibitor (Figure 4A). Thus, ATM kinase inhibition promotes RET/PTC rearrangements in human cells, even in the absence of an exogenous source of DSBs.

Next, we investigated how DNA-PK inhibition impacts endonuclease-induced DSB end-initiated RET/ $P T C$ rearrangements. It has previously been shown that

A

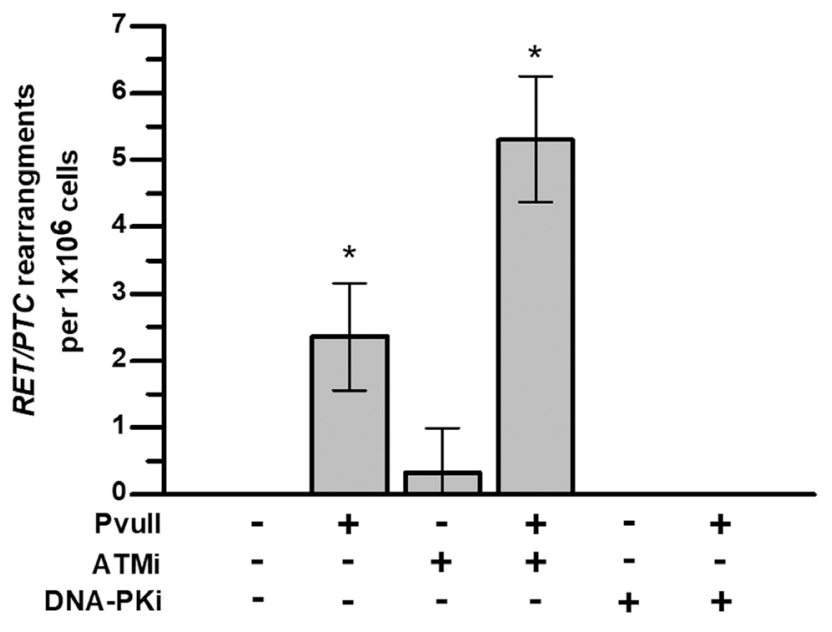

RET/PTC fusions occur at sites where there are 3-4 bp of sequence homology between the genes [23], which is characteristic of NHEJ. To test whether DNA-PK activity is required for the rearrangement to occur, HTori-3 cells were electroporated with PvuII restriction enzyme and treated with the pharmacologic DNA-PK inhibitor NU7441. No rearrangements was detected in cells treated with combination of PvuII and DNA-PK inhibitor or with DNAPK inhibitor alone (Figure 4A). This data, which is strikingly different to those obtained with the ATM kinase inhibitor, suggests that inhibition of DNA-PK, and therefore classical NHEJ, prevents the formation of RET/PTC rearrangements.

Finally, we investigated how inhibition of F-actin polymerization impacts endonuclease-induced DSB end-initiated RET/PTC rearrangements. For this, HTori-3 cells were transfected with vectors expressing either wild-type actin or G13R-mutated actin that is deficient in actin polymerization. PvuII-induced RET/ $P T C$ rearrangements were observed in $1.5 \pm 0.5$ cells per million in cells expressing wild-type actin, and in $4 \pm 1$ cells per million in cells expressing G13R-mutated actin (Figure 4B). The inhibition of F-actin polymerization in PvuII-electroporated HTori-3 human cells showed the tendency for the increase in frequency of RET/PTC rearrangements, however it did not reach the level of statistical significance.

\section{DISCUSSION}

In this study, we show that contact between homologous chromosomes after induction of DSBs in transcriptionally

B

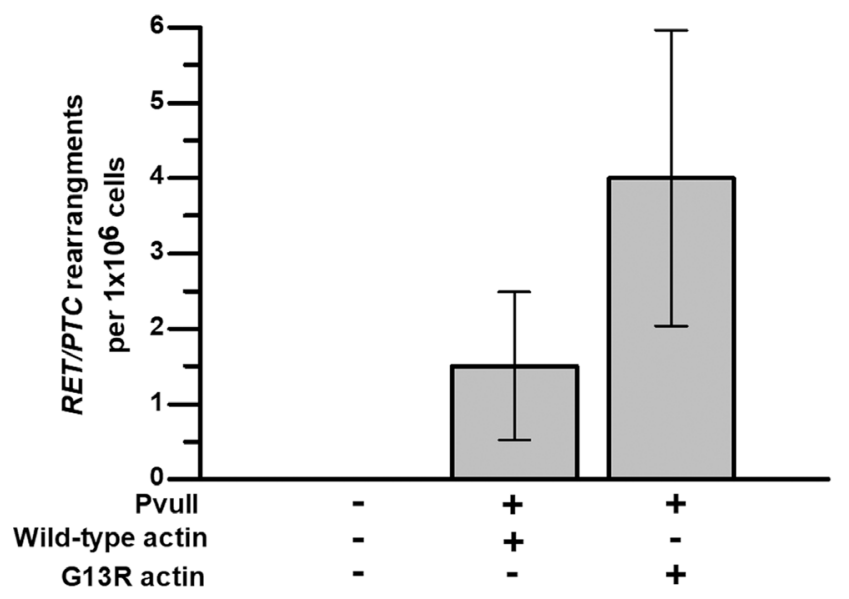

Figure 4: ATM activity and F-actin polymerization are required for chromosomal stability after induction of DSBs. HTori-3 cells were treated for $4 \mathrm{~h}$ with ATM kinase inhibitor KU55933 or DNA-PK inhibitor NU7441 immediately after electroporation with PvuII restriction endonuclease. (A) Graph showing the effect of ATM and DNA-PK inhibition on the formation of RET/PTC rearrangements in HTori-3 cells with and without PvuII-induced DNA DSBs. (B) Graph showing that F-actin polymerization deficiency after introduction of G13R-mutated actin increased the frequency of RET/PTC rearrangements in HTori-3 cells treated with PvuII endonuclease. Data are presented as means with $95 \% \mathrm{CI} ;{ }^{*}, P<0.05$. 
active chromatin in $\mathrm{G}_{0} / \mathrm{G}_{1}$-phase cells requires HDR proteins as well as nuclear myosin/actin motors. Furthermore, we demonstrate that ATM kinase inhibition or F-actin polymerization deficiency induce carcinogenic RET/PTC chromosome rearrangements in human cells.

In addition to our previous study where we reported the role of ATM kinase activity in homologous chromosomes contact [2], using 3D FISH and immunoFISH we demonstrate that BRCA1, RAD51 and RAD52 are also required to generate contact between homologous chromosomes after induction of DSBs in $\mathrm{G}_{0} / \mathrm{G}_{1}$-phase human cells. 3D FISH allows the direct enumeration of chromosomes and to exclude cells containing sister chromatids. In addition, 3D immuno-FISH with antiPCNA antibody identifies $\mathrm{G}_{0} / \mathrm{G}_{1}$-phase cells on the basis of pan-nuclear PCNA staining. Work in yeast predicts that HDR proteins will be recruited to a DSB end in $\mathrm{G}_{0} / \mathrm{G}_{1}$-phase cells before its mobility is increased and contact between homologous chromosomes is generated $[3,4]$. We found that RAD51 co-localizes with almost half of endonuclease-induced sites of contact between homologous chromosomes in $\mathrm{G}_{0} / \mathrm{G}_{1}$-phase cells. This suggest that the apical DNA damage signaling kinase- and HDR protein-dependent mechanism, that generates contact between homologous chromosomes after the induction of DSBs in $G_{0} / G_{1}$-phase cells, is conserved from yeast to human cells, although in yeast it is Mec1-dependent, and in humans it is ATM-dependent rather than ATR (Mec1)-dependent $[3,4]$. We have previously shown that ATM kinase activity and active transcription are required to generate contact between homologous chromosomes induced by DSBs in transcriptionally active chromatin outside of the nucleolus $[1,2]$. And it is consistent with the recent finding that ATM kinase activity is strictly required for the movement of DSBs in actively transcribed rDNA to the nucleolar periphery in $\mathrm{G}_{0} / \mathrm{G}_{1}$-phase human cells [13]. Thus, it appears that the mechanisms of DSBinduced mobility in transcribed rDNA and transcribed genes outside of the nucleolus are both dependent on ATM kinase and HDR proteins including RAD51.

A major mechanistic finding in this report, not been described in any other system to date, is that nuclear motors are required for contact between homologous chromosomes after the induction of DSBs in transcriptionally active regions, and these may play significant role in preserving genome integrity after DNA damage. Previous studies have shown that long-range directional movement of an interphase chromosome site is dependent on specific actin or nuclear myosin I (NMI) nuclear motors [14]. Our findings demonstrate that expression of mutant actin defective in polymerization as well as exposure to chemical inhibitors of nuclear motors, inhibits contact between homologous chromosomes after induction of DSBs in transcriptionally active chromatin.

To determine the functional significance of the ATM kinase- and nuclear motor-dependent mechanism that generates contact between homologous chromosomes after induction of DSBs in transcriptionally active chromatin, we enumerated carcinogenic chromosomal rearrangements using recently established assay that utilizes restriction enzymes to induce targeted DSBs in human thyroid cells HTori-3 [24]. Using this assay, we showed that ATM kinase activity is required for the repair of actively transcribed DNA, and ATM kinase inhibition can induce the RET/PTC carcinogenic rearrangements. Similarly, we observed that expression of mutant non-polymerizing actin, which also prevents homologous chromosomes contact, shows tendency to induce rearrangement in human cells. In summary, the results of this study reveal that contact between homologous chromosomes at the sites of DSBs in actively transcribed genes is dependent on HDR proteins and nuclear myosin/actin motors; functional ATM kinase and proper F-actin polymerization are required for prevention of carcinogenic chromosomal rearrangement generation upon induction of DNA DSBs in human cells.

\section{MATERIALS AND METHODS}

\section{Cell lines}

TPC-1 is a human cell line derived from a papillary thyroid carcinoma that expresses the RET/PTC1 oncogene. We have previously confirmed that $D A B 1$ and GRIPI chromosome loci studied here are diploid in interphase TPC-1 cells $[1,2]$. TPC1 cell line was propagated in DMEM media supplemented with $10 \%$ FBS. HTori-3 is a human normal thyroid cell line originally immortalized by SV40 transfection [26]. These cells are partially transformed but retain thyroid differentiation characteristics expressing sodium/iodide symporter and thyroglobulin [23, 24]. HTori-3 cell line was propagated in RPMI 1640 media supplemented with 10\% FBS.

\section{Induction of DNA DSBs by I-PpoI in TPC-1 cells}

To establish a cell line stably expressing I-PpoI, HA-ER-I-PpoI, retrovirus (gift of M. Kastan, Duke Cancer Institute, Durham, NC) was generated as described previously [27] and used to infect TPC-1 cells. Selection of TPC-1 cells expressing HA-ER-I-PpoI (TPC-1/I-PpoI) was done using supplementation of culture medium with $1 \mu \mathrm{g} / \mathrm{mL}$ puromycin for 2 weeks. To induce DNA DSBs, cells were treated with $1 \mu \mathrm{M}$ 4-hydroxytamoxifen (4-OHT) for $6 \mathrm{~h}[17,28]$. DSB induction was monitored by $\gamma \mathrm{H} 2 \mathrm{AX}$ immunofluorescence.

\section{$\gamma \mathrm{H} 2 \mathrm{AX}$ immunofluorescence}

Immunofluorescence staining for $\gamma \mathrm{H} 2 \mathrm{AX}$ was performed to confirm formation of I-PpoI- and PvuIIinduced DSBs in TPC-1/I-PpoI and Htori-3 cells, 
respectively. Cells cultured on coverslips were fixed with ice-cold $4 \%$ formaldehyde for $10 \mathrm{~min}$ followed by the permeabilization with $0.5 \%$ Triton $\mathrm{X}-100 / \mathrm{PBS}$ on ice for $5 \mathrm{~min}$. After washing with PBS, cells were stained with staining solution (20 mM Tris, pH7.6, $137 \mathrm{mMNaCl}, 10 \%$ skimmed milk, and $0.5 \%$ Tween-20) containing antiphosphorylated histone $\mathrm{H} 2 \mathrm{AX}$ primary antibody (Upstate Biotechnology, Buffalo, NY, USA) at 1:1000 dilution for $2 \mathrm{~h}$ at $37^{\circ} \mathrm{C}$. Coverslips were washed with PBS twice and incubated with Alexa488-labeled anti-mouse IgG (Molecular Probes, Eugene, OR, USA) in staining solution (1:200) for $1 \mathrm{~h}$ at $378 \mathrm{C}$. After washing with PBS, samples were counterstained with DAPI. Cells with more than five $\gamma \mathrm{H} 2 \mathrm{AX}$ foci per nucleus were considered positive for I-PpoI- or PvuII-induced cleavage.

\section{Analysis of contact between specific regions of homologous chromosomes using three- dimensional FISH and immuno-FISH}

To analyze homologous chromosomes contact, TPC/I-PpoI cells from each experiment were 3D fixed and processed to FISH with region-specific probes. Cells cultured in chamber slides from each experiment were subjected to 3D fixation with $4 \%$ paraformaldehyde for 10 minutes at $4{ }^{\circ} \mathrm{C}$, permeabilization on ice with $0.5 \%$ Triton X-100/PBS for 15 minutes followed by immersion into ice-cold 20\% Glycerol/PBS for $30 \mathrm{~min}$ and 4-6 times freeze-thaw treatment in liquid nitrogen [29]. For FISH, $3 \mathrm{D}$ fixed slides were washed in $0,05 \%$ Triton X-100/PBS for $15 \mathrm{~min}$ at room temperature (RT) and pretreated with $100 \mathrm{ug} / \mathrm{ul}$ RNase A in $1 \mathrm{mM}$ EDTA/PBS for 1 hour at $37^{\circ} \mathrm{C}$, followed by post-fixation with $2 \%$ paraformaldehyde for 5 minutes at RT. After washing in 2 XSCC slides were subjected to hybridization $\left(73^{\circ} \mathrm{C}-5\right.$ minutes $/ 37^{\circ} \mathrm{C}-14-$ 18 hours) with labeled probe in hybridization buffer (55\% formamide, 10\% dextran sulfate in 2xSCC, pH7.0). For FISH probes generation, RP11-328F17 + RP11393123 (DAB1 gene region), RP11-754J4 (GRIP1 gene region), $\mathrm{CH} 17-12 \mathrm{M} 21$ (5q15-q21 intergenic region) and RP11-513N24 (16q22 intergenic region) BAC clones (BAC/PAC Resources, Children's Hospital, Oakland) were labeled by nick translation using d-UTP tagged Spectrum dyes (Abbott Laboratories) according to manufacture instructions. Post hybridization washes were performed with $50 \%$ formamide $/ 2 \mathrm{xSSC}$ at $42^{\circ} \mathrm{C}$ and $2 \mathrm{XSCC}$ at $37^{\circ} \mathrm{C}$ followed by embedding with VECTASHIELD mounting medium with DAPI (Vector Laboratories).

Immuno-FISH, i.e. immunostaining followed by 3D FISH, was performed for the simultaneous detection of specific DNA targets and nuclear proteins [30, 31]. Mouse monoclonal antibodies for PCNA (Sigma-Aldrich, P-8825, 1:1500) and rabbit polyclonal antibody for RAD51 (Abcam, ab 63801, 1:100) were used. Briefly, cultured cells were fixed with ice-cold 4\% PFA for 10 minutes and permeabilized on ice with $0.5 \%$ Triton X-100/
PBS for 15 minutes followed by standard immunostaining with sequential incubations with primary and secondary antibodies. Immunostained slides were subjected to postfixation with $2 \%$ PFA for 10 minutes at RT, equilibration with 20\% Glycerol/PBS followed by 4-6 freeze-thaw cycles and FISH as described above.

Confocal microscopy was performed using a Leica SP5 TCS 4D confocal laser scanning fluorescence microscope using a $63 \times, 1.4$ N.A. oil PlanApo objective. For each experimental condition, at least 1,000 nuclei of TPC/I-PpoI $\mathrm{G}_{0} / \mathrm{G}_{1}$-phase cells were analyzed. We identified and evaluated $\mathrm{G}_{0} / \mathrm{G}_{1}$-phase cells on the basis of their morphology and the unambiguous identification of 2 copies of each gene or diffuse PCNA staining. Nuclear boundaries were identified by DAPI staining, and image stacks were acquired with z steps of $0.13 \mu \mathrm{m}$. The digital image stacks were reconstructed using Velocity software (PerkinElmer). Site-specific probes in each nucleus were identified by using the appropriate filter cube, and signals were scored as positive for contact when the space between them was smaller than size of one signal.

\section{siRNA gene silencing and Quantitative qRT-PCR}

Down-regulation of HDR pathway (BRCA1, $R A D 51, R A D 52)$, NHEJ pathway (KU70, DNA-PK, $L I G I V)$ and $N M I$ genes expression in TPC1/I-PpoI cells was performed using siRNA pools (Dharmacon) according to manufacturer instructions. Change in expression levels of studied genes were analyzed using qRT-PCR with CyberGreen (ABI Biosystems) 72 hours after treatment with siRNAs. Data of $P G K$ expression was used for normalization. Experiments were performed in triplicates.

\section{Chemical disruption of normal F-actin polymerization}

To alter F-actin polymerization in TPC-1/I-PpoI cells they were treated with $200 \mathrm{nM}$ Latrunculin A or 100nM Jasplakinolide for 2 hours after DSBs induction with 4-OHT. Experiments were performed in triplicates.

\section{Chemical inhibition of ATM and DNA-PK}

Htori-3 cells were treated with $10 \mu \mathrm{M}$ ATM kinase inhibitor KU55933 or $5 \mu \mathrm{M}$ DNA-PK inhibitor NU7441 for 4 hours immediately after electroporation with PvuII restriction enzyme. The efficacy of KU55933 and NU7441 in HTori-3 cells was determined by immunoblotting with anti-ATM (MAT3-4G10/8; SigmaAldrich), anti-phosphoserine-1981 ATM (EP1890Y; Epitomics), anti-DNA-PK (4062; Cell Signaling) and anti-phosphoserine-2056 (18192; Abcam) antibodies (data not shown). Experiments were performed in triplicates. 


\section{Actin plasmids}

Three expression plasmids (gift of Dr. A Belmont) coding different forms of actin were used: wild type actin, G13R actin that is deficient in F-actin polymerization and mutant S14C actin that increases F-actin polymerization $[14,22]$. TPC1/I-PpoI cells were transfected with wild type, G13R or S14C actin coding plasmids; Htori-3 cells were transfected with wild type or G13R actin coding plasmids using Lipofectamine 2000 (Invitrogen) according to manufacturer protocol. After 2 weeks of selection with $500 \mu \mathrm{g} / \mathrm{mL}$ of G418, cells were subjected to the experiments with DSBs induction.

\section{PvuII-induced $R E T / P T C$ rearrangements in Htori-3 cells}

Scheme of the detection of RET/PTC rearrangements after introduction of DNA DSBs by PvuII is illustrated in Supplementary Figure 2. For each experiment $2 \times 10^{6}$ of HTori- 3 cells were electroporated with $25 \mathrm{U}$ of PvuII restriction enzyme (New England Bio Labs, Ipswick, MA, USA) at $50 \mathrm{mC}$ charge (400 V and $125 \mathrm{mF}$ ) using Gene Pulser Xcell Electroporator (Bio$\mathrm{Rad}$ ) as previously described [32]. DSB induction was monitored by $\gamma \mathrm{H} 2 \mathrm{AX}$ immunofluorescence staining at $4 \mathrm{~h}$ post-electroporation. For the detection of PvuIIinduced RET/PTC rearrangements the electroporated cells were seeded in 30 T25 flasks $\left(6.6 \times 10^{4}\right.$ cells per flask). To sustain continuous growth, cells were transferred to T75 flasks and harvested when 90\% confluence was reached. RNA was extracted from each flask using a Trizol reagent (Invitrogen) and mRNA was purified using Oligotex mRNA mini kit (Qiagen). Detection of RET/PTC was performed using RT followed by PCR with primers for RET/PTC1 and RET/PTC3; and Southern blot hybridization of the PCR products with ${ }^{32} \mathrm{P}$-labeled oligonucleotide specific probes as described previously [23]. Evidence for RET/PTC3 or RET/PTC1 rearrangement in the cells from a given flask was scored as one $R E T / P T C$ event. The experiments were performed in triplicates.

\section{Statistical analysis}

Statistical analysis was performed using the IBM SPSS Statistics version 25 software package. Data were analyzed using $T$-test for two groups comparison or Oneway ANOVA followed by Tukey-Kramer posttest for multiple group comparison. All P values were 2-sided and considered significant if less than 0.05 . In the experiments on generation of RET/PTC, due to data structure, the comparisons between experimental groups were based on the estimates of $95 \%$ confidence intervals (CI). The differences between means that did not have an overlap of 95\% CI were considered significant.

\section{Abbreviations}

DSB: DNA double strand break; NHEJ: non homologous end-joining; HDR: homology-directed repair; ALT: alternative lengthening of telomeres; NMI: nuclear myosin I; FISH: fluorescence in situ hybridization; 4-OHT: 4-hydroxytamoxifen.

\section{Author contributions}

YEN designed and supervised all experiments. CJB contributed to the design of experiments. MG and VNE completed all technical aspects of the experiments. AVN contributed to data analysis. YEN, CJB and AVN wrote the manuscript.

\section{ACKNOWLEDGMENTS}

We thank M. Kastan (Duke Cancer Institute, Durham, NC) for providing HA-ER-I-PpoI retrovirus and Dr. A. Belmont (Department of Cell and Developmental Biology, University of Illinois, Urbana-Champaign, Urbana, IL) for providing wild type and mutant actin genes coding expression plasmids.

\section{CONFLICTS OF INTEREST}

None declared.

\section{FUNDING}

This work was supported by the National Institutes of Health [CA88041, CA204173, P30CA047904]. Funding for open access charge: National Institutes of Health.

\section{REFERENCES}

1. Gandhi M, Evdokimova VN, Cuenco KT, Bakkenist CJ, Nikiforov YE. Homologous chromosomes move and rapidly initiate contact at the sites of double-strand breaks in genes in G(0)-phase human cells. Cell Cycle. 2013; 12: 547-52. https://doi.org/10.4161/cc.23754.

2. Gandhi M, Evdokimova VN, T Cuenco K, Nikiforova MN, Kelly LM, Stringer JR, Bakkenist CJ, Nikiforov YE. Homologous chromosomes make contact at the sites of double-strand breaks in genes in somatic G0/G1-phase human cells. Proc Natl Acad Sci U S A. 2012; 109: 9454-9. https://doi.org/10.1073/pnas.1205759109.

3. Mine-Hattab J, Rothstein R. Increased chromosome mobility facilitates homology search during recombination. Nature Cell Biology. 2012; 14: 510-U136. https://doi.org/ Doi 10.1038/Ncb2472.

4. Dion V, Kalck V, Horigome C, Towbin BD, Gasser SM. Increased mobility of double-strand breaks requires $\mathrm{Mec} 1$, 
Rad9 and the homologous recombination machinery. Nat Cell Biol. 2012; 14: 502-9. https://doi.org/10.1038/ ncb2465.

5. Aten JA, Stap J, Krawczyk PM, van Oven CH, Hoebe RA, Essers J, Kanaar R. Dynamics of dna double-strand breaks revealed by clustering of damaged chromosome domains. Science. 2004; 303: 92-5. https://doi.org/10.1126/ science. 1088845 .

6. Difilippantonio S, Gapud E, Wong N, Huang CY, Mahowald G, Chen HT, Kruhlak MJ, Callen E, Livak F, Nussenzweig MC, Sleckman BP, Nussenzweig A. 53BP1 facilitates long-range DNA end-joining during $\mathrm{V}(\mathrm{D}) \mathrm{J}$ recombination. Nature. 2008; 456: 529-33. https://doi. org/10.1038/nature07476.

7. Dimitrova N, Chen YC, Spector DL, de Lange T. 53BP1 promotes non-homologous end joining of telomeres by increasing chromatin mobility. Nature. 2008; 456: 524-8. https://doi.org/10.1038/nature07433.

8. Kruhlak MJ, Celeste A, Dellaire G, Fernandez-Capetillo O, Muller WG, McNally JG, Bazett-Jones DP, Nussenzweig A. Changes in chromatin structure and mobility in living cells at sites of DNA double-strand breaks. J Cell Biol. 2006; 172: 823-34. https://doi.org/10.1083/jcb.200510015.

9. Nelms BE, Maser RS, MacKay JF, Lagally MG, Petrini JH. In situ visualization of DNA double-strand break repair in human fibroblasts. Science. 1998; 280: 590-2.

10. Soutoglou E, Dorn JF, Sengupta K, Jasin M, Nussenzweig A, Ried T, Danuser G, Misteli T. Positional stability of single double-strand breaks in mammalian cells. Nat Cell Biol. 2007; 9: 675-82. https://doi.org/10.1038/ncb1591.

11. Aymard F, Bugler B, Schmidt CK, Guillou E, Caron P, Briois S, Iacovoni JS, Daburon V, Miller KM, Jackson SP, Legube G. Transcriptionally active chromatin recruits homologous recombination at DNA double-strand breaks. Nat Struct Mol Biol. 2014; 21: 366-74. https://doi. org/10.1038/nsmb.2796.

12. Cho NW, Dilley RL, Lampson MA, Greenberg RA. Interchromosomal homology searches drive directional ALT telomere movement and synapsis. Cell. 2014; 159: 108-21. https://doi.org/10.1016/j.cell.2014.08.030.

13. van Sluis M, McStay B. A localized nucleolar DNA damage response facilitates recruitment of the homology-directed repair machinery independent of cell cycle stage. Genes Dev. 2015. https://doi.org/10.1101/gad.260703.115.

14. Chuang $\mathrm{CH}$, Carpenter AE, Fuchsova B, Johnson T, de Lanerolle P, Belmont AS. Long-range directional movement of an interphase chromosome site. Curr Biol. 2006; 16: 825-31. https://doi.org/10.1016/j.cub.2006.03.059.

15. Bakkenist CJ, Kastan MB. DNA damage activates ATM through intermolecular autophosphorylation and dimer dissociation. Nature. 2003; 421: 499-506. https://doi. org/10.1038/nature01368.

16. White JS, Choi S, Bakkenist CJ. Transient ATM kinase inhibition disrupts DNA damage-induced sister chromatid exchange. Sci Signal. 2010; 3: ra44. https://doi.org/10.1126/ scisignal.2000758.

17. Berkovich E, Monnat RJ Jr, Kastan MB. Roles of ATM and NBS1 in chromatin structure modulation and DNA doublestrand break repair. Nat Cell Biol. 2007; 9: 683-90.

18. Stoddard BL. Homing endonuclease structure and function. Q Rev Biophys. 2005; 38: 49-95. https://doi.org/10.1017/ S0033583505004063.

19. Monnat RJ Jr, Hackmann AF, Cantrell MA. Generation of highly site-specific DNA double-strand breaks in human cells by the homing endonucleases I-Ppoi and I-Crei. Biochem Biophys Res Commun. 1999; 255: 88-93. https:// doi.org/10.1006/bbrc.1999.0152.

20. Bubb MR, Senderowicz AM, Sausville EA, Duncan KL, Korn ED. Jasplakinolide, a cytotoxic natural product, induces actin polymerization and competitively inhibits the binding of phalloidin to F-actin. J Biol Chem. 1994; 269: 14869-71.

21. Spector I, Shochet NR, Kashman Y, Groweiss A. Latrunculins: novel marine toxins that disrupt microfilament organization in cultured cells. Science. 1983; 219: 493-5.

22. Posern G, Miralles F, Guettler S, Treisman R. Mutant actins that stabilise F-actin use distinct mechanisms to activate the SRF coactivator MAL. EMBO J. 2004; 23: 3973-83. https://doi.org/10.1038/sj.emboj.7600404.

23. Caudill CM, Zhu Z, Ciampi R, Stringer JR, Nikiforov YE. Dose-dependent generation of RET/PTC in human thyroid cells after in vitro exposure to gamma-radiation: A model of carcinogenic chromosomal rearrangement induced by ionizing radiation. J Clin Endocrinol Metab. 2005; 90: 2364-9. https://doi.org/10.1210/jc.2004-1811.

24. Evdokimova V, Gandhi M, Rayapureddi J, Stringer JR, Nikiforov YE. Formation of carcinogenic chromosomal rearrangements in human thyroid cells after induction of double-strand DNA breaks by restriction endonucleases. Endocr Relat Cancer. 2012; 19: 271-81. https://doi. org/10.1530/ERC-11-0314.

25. Nikiforov YE, Rowland JM, Bove KE, Monforte-Munoz H, Fagin JA. Distinct pattern of ret oncogene rearrangements in morphological variants of radiation-induced and sporadic thyroid papillary carcinomas in children. Cancer Res. 1997; 57: $1690-4$.

26. Lemoine NR, Mayall ES, Jones T, Sheer D, McDermid S, Kendall-Taylor P, Wynford-Thomas D. Characterisation of human thyroid epithelial cells immortalised in vitro by simian virus 40 DNA transfection. Br J Cancer. 1989; 60: 897-903.

27. Pear W. Transient transfection methods for preparation of high-titer retroviral supernatants. Curr Protoc Mol Biol. 2001; Chapter 9: Unit9 11. https://doi. org/10.1002/0471142727.mb0911s36.

28. Berkovich E, Monnat RJ Jr, Kastan MB. Assessment of protein dynamics and DNA repair following generation of DNA double-strand breaks at defined genomic sites. Nat Protoc. 2008; 3: 915-22. 
29. Cremer M, Grasser F, Lanctot C, Muller S, Neusser M, Zinner R, Solovei I, Cremer T. Multicolor 3D fluorescence in situ hybridization for imaging interphase chromosomes. Methods Mol Biol. 2008; 463: 205-39.

30. Gandhi M, Medvedovic M, Stringer JR, Nikiforov YE. Interphase chromosome folding determines spatial proximity of genes participating in carcinogenic RET/PTC rearrangements. Oncogene. 2006; 25: 2360-6.
31. Solovei I, Cremer M. (2010). 3D-FISH on cultured cells combined with immunostaining. In: Bridger JM and Volpi EV, eds. Fluorescence in situ hybridization (FISH): Protocols and Applications: Springer Science+Business Media).

32. Carney JP, Morgan WF. Induction of DNA double-strand breaks by electroporation of restriction enzymes into mammalian cells. Methods Mol Biol. 1999; 113: 465-71. https://doi.org/10.1385/1-59259-675-4:465. 